Article

\title{
Hamiltonian Structure, Symmetries and Conservation Laws for a Generalized (2 + 1)-Dimensional Double Dispersion Equation
}

\author{
Elena Recio *(D), Tamara M. Garrido (D), Rafael de la Rosa (D) and María S. Bruzón (D) \\ Department of Mathematics, Universidad de Cádiz, Puerto Real, 11510 Cádiz, Spain \\ * Correspondence: elena.recio@uca.es
}

Received: 15 July 2019; Accepted: 6 August 2019; Published: 9 August 2019

\begin{abstract}
This paper considers a generalized double dispersion equation depending on a nonlinear function $f(u)$ and four arbitrary parameters. This equation describes nonlinear dispersive waves in $2+1$ dimensions and admits a Lagrangian formulation when it is expressed in terms of a potential variable. In this case, the associated Hamiltonian structure is obtained. We classify all of the Lie symmetries (point and contact) and present the corresponding symmetry transformation groups. Finally, we derive the conservation laws from those symmetries that are variational, and we discuss the physical meaning of the corresponding conserved quantities.
\end{abstract}

Keywords: Lie symmetry; conservation law; double dispersion equation; Boussinesq equation

\section{Introduction}

A well-known equation that models the motion of long dispersive shallow water waves, which are propagated in both directions, is the Boussinesq equation [1,2], given by:

$$
u_{t t}=u_{x x}+b u_{x x x x}+\alpha\left(u^{2}\right)_{x x} .
$$

This equation is an integrable system, which is well-posed for $b=-1$ and ill-posed for $b=1$.

Many modified and generalized Boussinesq equations have been considered in the literature. A generalization of the Boussinesq equation depending on a nonlinearity power $p \neq 0$ consists of replacing in (1) the nonlinear term $\left(u^{2}\right)_{x x}$ by $\left(u^{p}\right)_{x x}$. The resulting equation is given by:

$$
u_{t t}=u_{x x}+a u_{x x x x}+\alpha\left(u^{p}\right)_{x x} .
$$

A modified Boussinesq equation is obtained by substituting the fourth-order term $u_{x x x x}$ in (1) by $u_{t t x x}$, yielding:

$$
u_{t t}=u_{x x}+a u_{t t x x}+\alpha\left(u^{2}\right)_{x x} .
$$

This equation is well-posed. In [3-8], the Cauchy problem and initial boundary value problem were considered for these equations and also for a double dispersion equation that unifies the previous equations and depends on a nonlinear function $f(u)$. This generalized double dispersion equation is given by:

$$
u_{t t}=u_{x x}+a u_{t t x x}+b u_{x x x x}+d u_{t x x}+(f(u))_{x x} .
$$

Another interesting variant of the Boussinesq equation is given by the sixth-order equation:

$$
u_{t t}=u_{x x}+a u_{x x x x x x}+b u_{x x x x}+\left(u^{3}\right)_{x x},
$$


and its generalization:

$$
u_{t t}=c u_{x x}+a u_{x x x x x x}+b u_{x x x x}+(f(u))_{x x},
$$

where $f(u)$ is a nonlinear function. These equations model long gravity-capillary surface waves with a short amplitude, propagating in both directions in shallow water. The Cauchy problem was considered for Equation (5) in [9], and the Hamiltonian formulation and a complete classification of Lie point symmetries and conservation laws were obtained for Equation (6) in [10].

Recently, a generalization of Equation (2) to two spatial dimensions, given by:

$$
u_{t t}=u_{x x}+b u_{x x x x}+\alpha\left(u^{p+1}\right)_{x x}+\beta u_{y y}, \quad p \neq 0
$$

was considered in [11], where the point symmetries, conservation laws, and line soliton solutions were derived.

In this work, we consider a $(2+1)$-dimensional generalization of the double dispersion Equation (4), given by:

$$
u_{t t}=u_{x x}+a u_{t t x x}+b u_{x x x x}+d u_{t x x}+(f(u))_{x x}+\beta u_{y y}
$$

where $f(u)$ is a nonlinear arbitrary function and $a, b, d$, and $\beta$ are arbitrary parameters. The 2D generalized double dispersion (2D gDD) family of Equations (8) unifies the previous Equations (1)-(4) and (7). Some $(2+1)$-dimensional equations in this family were considered in recent literature and were shown to admit interesting exact solutions such as line solitons and lump solutions [11-13].

For any given partial differential equation (PDE), symmetries are transformations that leave invariant the whole space of solutions of the equation. Symmetries can be used to obtain reductions and exact group-invariant solutions. These invariant solutions play a key role in the investigation of certain analytical properties, e.g., asymptotic and blow-up behavior. In addition, explicit solutions can be used to assess the accuracy and reliability of numerical solution methods. For a given PDE, all admitted Lie symmetries can be determined by applying the Lie method.

A conservation law of a given evolution equation is a continuity equation that yields basic conserved quantities for all solutions. Some important uses, among others, are that they allow detecting and constructing mappings of nonlinear evolution equations to linear equations. Moreover, they can be used for studying integrability.

In Section 2, we write the 2D gDD Equation (8) as a potential equation, and we find a Lagrangian formulation for $d=0$. The associated Hamiltonian formulation is also included in Section 2. By using the Lie method, in Section 3, we classify all point and contact symmetries of the potential equation, and we include the corresponding symmetry groups. We provide an Appendix A with a summary of the computations. In Section 4, from the previous classification of Lie symmetries of the potential equation, the variational symmetries are found. Next, in Section 5, we derive the conservation laws of the potential equation from the variational symmetries by using Noether's theorem. Furthermore, we discuss the physical meaning of the associated conserved quantities. Finally, in Section 6, we give some conclusions.

\section{Potential Form and Hamiltonian Formulation}

The generalized double dispersion Equation (8) can be expressed in potential form by using a potential:

$$
u=v_{x}
$$

The resulting equation is then given by:

$$
G=v_{t t}-v_{x x}-a v_{t t x x}-b v_{x x x x}-d v_{t x x}-\left(f\left(v_{x}\right)\right)_{x}-\beta v_{y y}=0 .
$$


This potential Equation (10) admits a local Lagrangian structure:

$$
\frac{\delta L}{\delta v}=0
$$

if and only if $d=0$, where $\delta / \delta v$ is the variational derivative with respect to $v$ :

$$
\begin{aligned}
\frac{\delta}{\delta v}= & \partial_{v}-D_{t} \partial_{v_{t}}-D_{x} \partial_{v_{x}}-D_{y} \partial_{v_{y}}+D_{t}^{2} \partial_{v_{t t}}+D_{x}^{2} \partial_{v_{x x}}+D_{y}^{2} \partial_{v_{y y}}+D_{t} D_{x} \partial_{v_{t x}}+D_{t} D_{y} \partial_{v_{t y}} \\
& +D_{x} D_{y} \partial_{v_{x y}}+\cdots
\end{aligned}
$$

Indeed, the double dispersion equation in potential form (10) can be written as the Euler-Lagrangian equation of a local Lagrangian if and only if the Helmholtz conditions are satisfied [14,15], i.e., the Fréchet derivative of Equation (10) is self-adjoint. We recall [14,15] that the Fréchet derivative of a differential function $G\left(t, x, y, v, v_{t}, v_{x}, v_{y}, \ldots\right)$ acting on $P(t, x, y)$ is given by:

$$
\begin{aligned}
\delta_{P} G= & P \frac{\partial G}{\partial v}+D_{t} P \frac{\partial G}{\partial v_{t}}+D_{x} P \frac{\partial G}{\partial v_{x}}+D_{y} P \frac{\partial G}{\partial v_{y}}+D_{t}^{2} P \frac{\partial G}{\partial v_{t t}}+D_{x}^{2} P \frac{\partial G}{\partial v_{x x}}+D_{y}^{2} P \frac{\partial G}{\partial v_{y y}} \\
& +D_{t} D_{x} P \frac{\partial G}{\partial v_{t x}}+D_{t} D_{y} P \frac{\partial G}{\partial v_{t y}}+D_{x} D_{y} P \frac{\partial G}{\partial v_{x y}}+\cdots,
\end{aligned}
$$

and the adjoint Fréchet derivative is given by:

$$
\begin{aligned}
\delta_{P}^{*} G= & P \frac{\partial G}{\partial v}-D_{t}\left(P \frac{\partial G}{\partial v_{t}}\right)-D_{x}\left(P \frac{\partial G}{\partial v_{x}}\right)-D_{y}\left(P \frac{\partial G}{\partial v_{y}}\right)+D_{t}^{2}\left(P \frac{\partial G}{\partial v_{t t}}\right)+D_{x}^{2}\left(P \frac{\partial G}{\partial v_{x x}}\right) \\
& +D_{y}^{2}\left(P \frac{\partial G}{\partial v_{y y}}\right)+D_{t} D_{x}\left(P \frac{\partial G}{\partial v_{t x}}\right)+D_{t} D_{y}\left(P \frac{\partial G}{\partial v_{t y}}\right)+D_{x} D_{y}\left(P \frac{\partial G}{\partial v_{x y}}\right)-\cdots
\end{aligned}
$$

To show that the Fréchet derivative of the potential 2D gDD Equation (10) is self-adjoint, we compute the Fréchet derivative of Equation (10):

$$
\delta_{P} G=-f^{\prime \prime}\left(v_{x}\right) v_{x x} D_{x} P+D_{t}^{2} P-\left(1+f^{\prime}\left(v_{x}\right)\right) D_{x}^{2} P-\beta D_{y}^{2} P-d D_{t} D_{x}^{2} P-a D_{t}^{2} D_{x}^{2} P-b D_{x}^{4} P,
$$

and the adjoint Fréchet derivative of Equation (10):

$$
\delta_{P}^{*} G=D_{x}\left(f^{\prime \prime}\left(v_{x}\right) v_{x x} P\right)+D_{t}^{2} P-D_{x}^{2}\left(\left(1+f^{\prime}\left(v_{x}\right)\right) P\right)-\beta D_{y}^{2} P+d D_{t} D_{x}^{2} P-a D_{t}^{2} D_{x}^{2} P-b D_{x}^{4} P,
$$

where $P=P(t, x, y)$, and we verify that Expression (15) coincides with its adjoint (16) for all $P(t, x, y)$. It is straightforward to show that this only occurs iff $d=0$.

There are two main implications when a PDE admits a Lagrangian structure: the existence of conserved energy, momentum, etc., from Noether's theorem and the existence of a Hamiltonian formulation. From the physical point of view, the restriction $d=0$ on Equation (10) means that the dynamics is being restricted such that dissipative processes are being removed, leading to conservation of energy, momentum, etc.

The Lagrangian corresponding to the Lagrangian formulation (11) of Equation (10) is then given by:

$$
L=-\frac{1}{2} v_{t}^{2}+\frac{1}{2} v_{x}^{2}-\frac{1}{2} a v_{t t} v_{x x}-\frac{1}{2} b v_{x x}^{2}+F\left(v_{x}\right)+\frac{1}{2} \beta v_{y^{\prime}}^{2}
$$

where $F^{\prime}\left(v_{x}\right)=f\left(v_{x}\right)$.

The potential Equation (10) can be expressed as an equivalent evolution system, given by:

$$
\begin{aligned}
& v_{t}=w, \\
& w_{t}=v_{x x}+a v_{t t x x}+b v_{x x x x}+d v_{t x x}+f^{\prime}\left(v_{x}\right) v_{x x}+\beta v_{y y} .
\end{aligned}
$$


When $d=0$, the Lagrangian structure (11) yields a Hamiltonian formulation for the potential system (18), given by:

$$
\left(\begin{array}{c}
v \\
w
\end{array}\right)_{t}=J\left(\begin{array}{l}
\delta H / \delta v \\
\delta H / \delta w
\end{array}\right), \quad J=\left(\begin{array}{cc}
0 & 1 \\
-1 & 0
\end{array}\right)
$$

with the Hamiltonian density given by:

$$
H=\int_{\mathbb{R}^{2}}\left(\frac{1}{2} w^{2}+\frac{1}{2} v_{x}^{2}-\frac{1}{2} a v_{t t} v_{x x}-\frac{1}{2} b v_{x x}^{2}+F\left(v_{x}\right)+\frac{1}{2} \beta v_{y}^{2}\right) d x d y,
$$

where $F^{\prime}\left(v_{x}\right)=f\left(v_{x}\right)$ and $J$ is the Hamiltonian operator [15]. There exists an equivalent Hamiltonian structure in terms of the variable $u$, with the Hamiltonian given by a nonlocal expression in $u$ and $w$. By noting that $u_{t}=v_{t x}=w_{x}$ and by applying the variational derivative identity $\delta H / \delta v=-D_{x}(\delta H / \delta u)$, the Hamiltonian formulation is then given by:

$$
\left(\begin{array}{c}
u \\
w
\end{array}\right)_{t}=\mathcal{D}\left(\begin{array}{l}
\delta H / \delta u \\
\delta H / \delta w
\end{array}\right), \quad \mathcal{D}=\left(\begin{array}{cc}
0 & D_{x} \\
D_{x} & 0
\end{array}\right)
$$

where $\mathcal{D}$ is the Hamiltonian operator [15].

Note that when $a=0$ and $f\left(v_{x}\right)=v_{x}^{p+1}$, the generalized $(2+1)$-dimensional Boussinesq with $p$-power nonlinearity is obtained. The Hamiltonian structure for this equation was obtained in [11]. In addition, if $\beta=0$ and $p=1$, this Hamiltonian formulation is one of the Hamiltonian structures [15] of the ordinary Boussinesq Equation (1). In this section, these Hamiltonian structures have been extended for a more general $(2+1)$-dimensional double dispersion equation depending on an arbitrary function.

\section{Lie Symmetries}

For nonlinear evolution equations, symmetries are important since they can be used to determine groups of transformations, which leave the solution space of the equation invariant, and also because they lead to reductions and exact invariant solutions. The Hamiltonian structure derived in the previous section motivates studying the symmetries of the 2D gDD equation in potential form (10). The Lie symmetries of Equation (10) consist of point symmetries and contact symmetries, since the equation involves only a single dependent variable $v$ [16].

\subsection{Point Symmetries}

An infinitesimal point symmetry of the potential 2D gDD Equation (10) is a vector field of the form:

$$
\mathbf{X}=\tau(t, x, y, v) \partial_{t}+\xi^{x}(t, x, y, v) \partial_{x}+\xi^{y}(t, x, y, v) \partial_{y}+\eta(t, x, y, v) \partial_{v}
$$

whose prolongation leaves invariant the whole solution space of the equation,

$$
\left.\operatorname{pr} \mathbf{X}\left(v_{t t}-v_{x x}-a v_{t t x x}-b v_{x x x x}-d v_{t x x}-f^{\prime}\left(v_{x}\right) v_{x x}-\beta v_{y y}\right)\right|_{G=0}=0 .
$$

A point symmetry (22) of the potential 2D gDD Equation (10) generates a one-parameter Lie group of point transformations acting on dependent and independent variables that carries solutions of the equation into other solutions. This point symmetry transformation is then given by:

$$
\begin{aligned}
& \tilde{t}=t+\epsilon \tau(t, x, y, v)+O\left(\epsilon^{2}\right), \\
& \tilde{x}=x+\epsilon \tilde{\zeta}^{x}(t, x, y, v)+O\left(\epsilon^{2}\right), \\
& \tilde{y}=y+\epsilon \xi^{y}(t, x, y, v)+O\left(\epsilon^{2}\right), \\
& \tilde{v}=v+\epsilon \eta(t, x, y, v)+O\left(\epsilon^{2}\right),
\end{aligned}
$$


with $\epsilon$ being the group parameter. The action of a point symmetry on solutions of the potential 2D gDD Equation (10) yields:

$$
\begin{aligned}
v(t, x, y) \rightarrow \tilde{v}(t, x, y)= & v(t, x, y)+\epsilon\left(\eta \left(t, x, y, v(t, x, y)-\tau(t, x, y, v(t, x, y)) v_{t}(t, x, y)\right.\right. \\
& \left.-\eta^{x}(t, x, y, v(t, x, y)) v_{x}(t, x, y)-\eta^{y}(t, x, y, v(t, x, y)) v_{y}(t, x, y)\right)+O\left(\epsilon^{2}\right),
\end{aligned}
$$

corresponding to the characteristic form of the generator, given by:

$$
\hat{\mathbf{X}}=P \partial_{v}, \quad P=\eta-\tau v_{t}-\xi^{x} v_{x}-\xi^{y} v_{y}
$$

where $P$ is the symmetry characteristic. The invariance condition is then equivalently expressed in terms of the Fréchet derivative (13) of the potential 2D gDD Equation (10) acting on the symmetry characteristic $P$ as:

$$
0=\left.\operatorname{pr} \hat{\mathbf{X}}(G)\right|_{G=0}=\left.\delta_{P} G\right|_{G=0},
$$

yielding:

$$
D_{t}^{2} P-f^{\prime \prime}\left(v_{x}\right) v_{x x} D_{x} P-\left(1+f^{\prime}\left(v_{x}\right)\right) D_{x}^{2} P-\beta D_{y}^{2} P-d D_{t} D_{x}^{2} P-a D_{t}^{2} D_{x}^{2} P-b D_{x}^{4} P=0,
$$

which holds for all solutions of the 2D gDD equation in potential form (10), and is called the symmetry-determining equation of PDE (10). The determining Equation (28) splits with respect to the differential consequences of $v$ and leads to an overdetermined system of equations for $P, f\left(v_{x}\right)$, $a, b, d$, and $\beta$. Furthermore, we will also impose the classification conditions $f^{\prime \prime}\left(v_{x}\right) \neq 0$, which implies that the equation is nonlinear; $a^{2}+b^{2} \neq 0$ and $\beta \neq 0$, which respectively imply that the equation is a fourth-order PDE, and generalizes the $1 \mathrm{D}$ gDD Equation (4) to two spatial dimensions. We set up and solve the resulting determining system by using Maple, in particular the "rifsimp" and "pdsolve" commands. A summary of the steps followed for this computation is included in Appendix A. Therefore, we have the following classification result.

Theorem 1. (i) The point symmetries admitted by the $2 D$ generalized double dispersion potential Equation (10) for arbitrary $a, b, d, \beta$, and $f\left(v_{x}\right)$ with the conditions $a^{2}+b^{2} \neq 0, \beta \neq 0$, and $f^{\prime \prime}\left(v_{x}\right) \neq 0$ are generated by the transformations:

$$
\begin{aligned}
& \mathbf{X}_{1}=\partial_{t} \\
& (\tilde{t}, \tilde{x}, \tilde{y}, \tilde{v})_{1}=(t+\epsilon, x, y, v), \quad \text { time-translation. } \\
& \mathbf{X}_{2}=\partial_{x} \\
& (\tilde{t}, \tilde{x}, \tilde{y}, \tilde{v})_{2}=(t, x+\epsilon, y, v), \quad \text { space-translation. } \\
& \mathbf{X}_{3}=\partial_{y} \\
& (\tilde{t}, \tilde{x}, \tilde{y}, \tilde{v})_{3}=(t, x, y+\epsilon, v), \quad \text { space-translation. } \\
& \mathbf{X}_{4, g, h}=(g(y+\sqrt{\beta} t)+h(y-\sqrt{\beta} t)) \partial_{v}, \\
& (\tilde{t}, \tilde{x}, \tilde{y}, \tilde{v})_{4}=(t, x, y, v+(g(y+\sqrt{\beta} t)+h(y-\sqrt{\beta} t)) \epsilon) .
\end{aligned}
$$

The last symmetry is a linear combination of two infinite-dimensional families, with $g(y+\sqrt{\beta} t)+h(y-$ $\sqrt{\beta} t)$ being the general solution of the linear equation $P_{t t}-\beta P_{y y}=0$ for $P=P(t, y)$.

(ii) The 2D generalized double dispersion potential Equation (10) admits additional point symmetries for special $f\left(v_{x}\right), a, b$, or $d$, in the following cases: 
(a) $a=0, d=0$, arbitrary $f\left(v_{x}\right), b$, and $\beta$,

$$
\begin{aligned}
& \mathbf{X}_{5}=y \partial_{t}+\beta t \partial_{y}, \\
& (\tilde{t}, \tilde{x}, \tilde{y}, \tilde{v})_{5}=\left(\cosh (\epsilon \sqrt{\beta}) t+\frac{1}{\sqrt{\beta}} \sinh (\epsilon \sqrt{\beta}) y, x, \cosh (\epsilon \sqrt{\beta}) y+\sqrt{\beta} \sinh (\epsilon \sqrt{\beta}) t, v\right), \\
& \quad \text { boost in the plane }(y, t) .
\end{aligned}
$$

(b) $f\left(v_{x}\right)=\alpha\left(v_{x}+c\right)^{p+1}-v_{x}, a=0$, arbitrary $b, d$, and $\beta$,

$$
\begin{aligned}
& \mathbf{X}_{6}=2 p t \partial_{t}+p x \partial_{x}+2 p y \partial_{y}+((p-2) v-2 c x) \partial_{v} \\
& (\tilde{t}, \tilde{x}, \tilde{y}, \tilde{v})_{6}=\left(e^{2 p \epsilon} t, e^{p \epsilon} x, e^{2 p \epsilon} y, e^{(p-2) \epsilon} v-2 c x \epsilon\right), \quad \text { scaling and shift. }
\end{aligned}
$$

(c) $f\left(v_{x}\right)=\frac{1}{\alpha} \ln \left(\alpha\left(v_{x}+c\right)\right)-v_{x}, a=0$, arbitrary $b, d$, and $\beta$,

$$
\begin{aligned}
& \mathbf{X}_{7}=2 t \partial_{t}+x \partial_{x}+2 y \partial_{y}+(3 v+2 c x) \partial_{v} \\
& (\tilde{t}, \tilde{x}, \tilde{y}, \tilde{v})_{7}=\left(e^{2 \epsilon} t, e^{\epsilon} x, e^{2 \epsilon} y, e^{3 \epsilon} v+2 c x \epsilon\right), \quad \text { scaling and shift. }
\end{aligned}
$$

(d) $f\left(v_{x}\right)=\alpha\left(v_{x}+c\right)^{p+1}-v_{x}, b=0, d=0$, arbitrary a and $\beta$,

$$
\begin{aligned}
& \mathbf{X}_{8}=p t \partial_{t}+p y \partial_{y}-2(v+c x) \partial_{v} \\
& (\tilde{t}, \tilde{x}, \tilde{y}, \tilde{v})_{8}=\left(e^{p \epsilon} t, x, e^{p \epsilon} y, e^{-2 \epsilon} v-2 c x \epsilon\right), \quad \text { scaling and shift. }
\end{aligned}
$$

(e) $f\left(v_{x}\right)=\alpha e^{p v_{x}}-v_{x}, b=0, d=0$, arbitrary $a$ and $\beta$,

$$
\begin{aligned}
& \mathbf{X}_{9}=p t \partial_{t}+p y \partial_{y}-2 x \partial_{v} \\
& (\tilde{t}, \tilde{x}, \tilde{y}, \tilde{v})_{9}=\left(e^{p \epsilon} t, x, e^{p \epsilon} y, v-2 x \epsilon\right), \quad \text { scaling and shift. }
\end{aligned}
$$

The classification of the maximal point symmetry Lie algebras for the 2D gDD potential Equation (10) is shown in the following theorem. For each case, the basis of generators and its non-zero Lie brackets are included.

Theorem 2. The 2D generalized double dispersion potential Equation (10) admits the maximal symmetry algebras (with corresponding non-zero commutator structure) given by:

(i) arbitrary $f\left(v_{x}\right), a, b, d$, and $\beta$,

$$
\begin{gathered}
\mathbf{X}_{1}, \mathbf{X}_{2}, \mathbf{X}_{3}, \mathbf{X}_{4, g, h} ; \\
{\left[\mathbf{X}_{1}, \mathbf{X}_{4, g, h}\right]=\mathbf{X}_{4, \sqrt{\beta} g^{\prime},-\sqrt{\beta} h^{\prime}} ; \quad\left[\mathbf{X}_{3}, \mathbf{X}_{4, g, h}\right]=\mathbf{X}_{4, g^{\prime}, h^{\prime}} .}
\end{gathered}
$$

(ii) $a=0, d=0$, arbitrary $f\left(v_{x}\right), b$, and $\beta$,

$$
\begin{gathered}
\mathbf{X}_{1}, \mathbf{X}_{2}, \mathbf{X}_{3}, \mathbf{X}_{4, g, h}, \mathbf{X}_{5} ; \\
{\left[\mathbf{X}_{1}, \mathbf{X}_{5}\right]=\beta \mathbf{X}_{3} ; \quad\left[\mathbf{X}_{3}, \mathbf{X}_{5}\right]=\mathbf{X}_{1} ; \quad\left[\mathbf{X}_{4, g, h}, \mathbf{X}_{5}\right]=\mathbf{X}_{4, g_{1}, h_{1}},}
\end{gathered}
$$

where $g_{1}=\sqrt{\beta}(y+\sqrt{\beta} t) g^{\prime}$ and $h_{1}=-\sqrt{\beta}(y-\sqrt{\beta} t) h^{\prime}$.

(iii) $f\left(v_{x}\right)=\alpha\left(v_{x}+c\right)^{p+1}-v_{x}, a=0$, arbitrary $b, d$, and $\beta$,

$$
\begin{gathered}
\mathbf{X}_{1}, \mathbf{X}_{2}, \mathbf{X}_{3}, \mathbf{X}_{4, g, h}, \mathbf{X}_{6} ; \\
{\left[\mathbf{X}_{1}, \mathbf{X}_{6}\right]=2 p \mathbf{X}_{1} ; \quad\left[\mathbf{X}_{2}, \mathbf{X}_{6}\right]=p \mathbf{X}_{2}+\mathbf{X}_{4,-2 c, 0} ; \quad\left[\mathbf{X}_{3}, \mathbf{X}_{6}\right]=2 p \mathbf{X}_{3} ; \quad\left[\mathbf{X}_{4, g, h}, \mathbf{X}_{6}\right]=\mathbf{X}_{4, g_{2}, h_{2}},}
\end{gathered}
$$
where $g_{2}=(p-2) g-2 p(y+\sqrt{\beta} t) g^{\prime}$ and $h_{2}=(p-2) h-2 p(y-\sqrt{\beta} t) h^{\prime}$. 
(iv)

$f\left(v_{x}\right)=\frac{1}{\alpha} \ln \left(\alpha\left(v_{x}+c\right)\right)-v_{x}, a=0$, arbitrary $b, d$, and $\beta$,

$$
\begin{gathered}
\mathbf{X}_{1}, \mathbf{X}_{2}, \mathbf{X}_{3}, \mathbf{X}_{4, g, h}, \mathbf{X}_{7} ; \\
{\left[\mathbf{X}_{1}, \mathbf{X}_{7}\right]=2 \mathbf{X}_{1} ; \quad\left[\mathbf{X}_{2}, \mathbf{X}_{7}\right]=\mathbf{X}_{2}+\mathbf{X}_{4,2 c, 0} ; \quad\left[\mathbf{X}_{3}, \mathbf{X}_{7}\right]=2 \mathbf{X}_{3} ; \quad\left[\mathbf{X}_{4, g, h}, \mathbf{X}_{7}\right]=\mathbf{X}_{4, g_{3}, h_{3},}} \\
\text { where } g_{3}=3 g-2(y+\sqrt{\beta} t) g^{\prime} \text { and } h_{3}=3 h-2(y-\sqrt{\beta} t) h^{\prime} . \\
\text { (v) } f\left(v_{x}\right)=\alpha\left(v_{x}+c\right)^{p+1}-v_{x}, b=0, d=0, \text { arbitrary a and } \beta,
\end{gathered}
$$

$$
\begin{gathered}
\mathbf{X}_{1}, \mathbf{X}_{2}, \mathbf{X}_{3}, \mathbf{X}_{4, g, h}, \mathbf{X}_{8} ; \\
{\left[\mathbf{X}_{1}, \mathbf{X}_{8}\right]=p \mathbf{X}_{1} ; \quad\left[\mathbf{X}_{2}, \mathbf{X}_{8}\right]=\mathbf{X}_{4,-2 c, 0} ; \quad\left[\mathbf{X}_{3}, \mathbf{X}_{8}\right]=p \mathbf{X}_{3} ; \quad\left[\mathbf{X}_{4, g, h}, \mathbf{X}_{8}\right]=\mathbf{X}_{4, g_{4}, h_{4}},}
\end{gathered}
$$$$
\text { where } g_{4}=-2 g-p(y+\sqrt{\beta} t) g^{\prime} \text { and } h_{4}=-2 h-p(y-\sqrt{\beta} t) h^{\prime} \text {. }
$$

(vi) $f\left(v_{x}\right)=\alpha e^{p v_{x}}-v_{x}, b=0, d=0$, arbitrary $a$ and $\beta$,

$$
\begin{gathered}
\mathbf{X}_{1}, \mathbf{X}_{2}, \mathbf{X}_{3}, \mathbf{X}_{4, g, h}, \mathbf{X}_{9} ; \\
{\left[\mathbf{X}_{1}, \mathbf{X}_{9}\right]=p \mathbf{X}_{1} ; \quad\left[\mathbf{X}_{2}, \mathbf{X}_{9}\right]=\mathbf{X}_{4,-2,0} ; \quad\left[\mathbf{X}_{3}, \mathbf{X}_{9}\right]=p \mathbf{X}_{3} ; \quad\left[\mathbf{X}_{4, g, h}, \mathbf{X}_{9}\right]=\mathbf{X}_{4, g_{5}, h_{5}},}
\end{gathered}
$$

where $g_{5}=-p(y+\sqrt{\beta} t) g^{\prime}$ and $h_{5}=-p(y-\sqrt{\beta} t) h^{\prime}$.

(vii) $f\left(v_{x}\right)=\alpha\left(v_{x}+c\right)^{p+1}-v_{x}, a=0, d=0$, arbitrary $b$ and $\beta$,

$$
\mathbf{X}_{1}, \mathbf{X}_{2}, \mathbf{X}_{3}, \mathbf{X}_{4, g, h}, \mathbf{X}_{5}, \mathbf{X}_{6}
$$

$$
f\left(v_{x}\right)=\frac{1}{\alpha} \ln \left(\alpha\left(v_{x}+c\right)\right)-v_{x}, a=0, d=0, \text { arbitrary } b \text { and } \beta,
$$

$$
\mathbf{X}_{1}, \mathbf{X}_{2}, \mathbf{X}_{3}, \mathbf{X}_{4, g, h}, \mathbf{X}_{5}, \mathbf{X}_{7}
$$

For arbitrary $f\left(v_{x}\right), a, b, d$, and $\beta$, the 2D gDD potential Equation (10) admits a four-dimensional Lie algebra consisting of time-translation symmetry (29), space-translation symmetries (29) and (30), and infinite-dimensional symmetry families (32). When $a=0$ and $d=0$, Equation (10) becomes a 2D generalized Boussinesq equation in potential form and admits a five-dimensional algebra that includes the previous symmetries and also a boost in the plane $(y, t)(33)$. The classification of point symmetries was already known for the 2D Boussinesq Equation (7) in potential form when $f\left(v_{x}\right)=v_{x}^{p+1}$ [11]. However, we remark that this maximal algebra is also admitted by Equation (10) with $a=0$ and $d=0$ for any $f\left(v_{x}\right)$.

We also note that for each of the cases (iii)-(viii) of Theorem 2, the specific forms of the function $f\left(v_{x}\right)$ will result in the dropping of the second-order term $v_{x x}$ in the 2D gDD potential Equation (10). Therefore, the resulting equations are not properly Boussinesq-type equations, but some other fourth-order nonlinear dispersive PDEs in $2+1$ dimensions that admit five-dimensional algebras consisting of time and space-translation symmetries, infinite symmetry families, plus a scaling and shift symmetry. In particular, when $a=0$ and $d=0$ and for the specific forms of $f\left(v_{x}\right)$ in Cases (vii) and (viii), the equations are respectively given by:

$$
v_{t t}-b v_{x x x x}-\alpha(p+1)\left(v_{x}+c\right)^{p} v_{x x}-\beta v_{y y}=0
$$

and:

$$
v_{t t}-b v_{x x x x}-\frac{1}{\alpha\left(v_{x}+c\right)} v_{x x}-\beta v_{y y}=0 .
$$

Both equations admit six-dimensional algebras consisting of time and space-translation symmetries, infinite symmetry families, boost symmetry, and scaling-shift symmetry. 


\subsection{Contact Symmetries}

A contact symmetry of the potential 2D gDD Equation (10) is a one-parameter Lie group of transformations that leaves invariant the solution space of the equation and in which the transformation of $(t, x, y, v)$ essentially depends on $v_{t}, v_{x}$, or $v_{y}$. The corresponding symmetry generator is given by:

$$
\mathbf{X}=\tau \partial_{t}+\xi^{x} \partial_{x}+\xi^{y} \partial_{y}+\eta \partial_{v}+\eta^{t} \partial_{v_{t}}+\eta^{x} \partial_{v_{x}}+\eta^{y} \partial_{v_{y}}
$$

and the characteristic form of this generator is then given by:

$$
\hat{\mathbf{X}}=P\left(t, x, y, v, v_{t}, v_{x}, v_{y}\right) \partial_{v},
$$

with:

$$
\begin{aligned}
& \tau=-P_{v_{t}}, \quad \xi^{x}=-P_{v_{x}}, \quad \xi^{y}=-P_{v_{y}}, \quad \eta=P-v_{t} P_{v_{t}}-v_{x} P_{v_{x}}-v_{y} P_{v_{y}}, \\
& \eta^{t}=P_{t}+v_{t} P_{v}, \quad \eta^{x}=P_{x}+v_{x} P_{v}, \quad \eta^{y}=P_{y}+v_{y} P_{v} .
\end{aligned}
$$

A contact symmetry yields a prolonged point symmetry iff the symmetry characteristic $P$ is at most linear in $v_{t}, v_{x}$, and $v_{y}$.

The invariance of the potential 2D gDD Equation (10) under the contact symmetry transformation is expressed by the determining Equation (28) holding for all solutions $v(t, x, y)$ of Equation (10). Then, the determining equation splits into an overdetermined system of equations for $P\left(t, x, y, v, v_{t}, v_{x}, v_{y}\right)$ together with the function $f\left(v_{x}\right)$ and the parameters of the equation $a, b, d$, and $\beta$. We impose again the classification conditions $f^{\prime \prime}\left(v_{x}\right) \neq 0, a^{2}+b^{2} \neq 0$ and $\beta \neq 0$. We set up and solve this system by using the Maple "rifsimp" and "pdsolve" commands. This computation is analogously done by using the steps outlined in Appendix A for the Lie point symmetries, but considering in this case the contact symmetry in characteristic form (41). Therefore, we obtain the following result.

Theorem 3. The 2D generalized double dispersion potential Equation (10) does not admit any contact symmetry except for those that reduce to prolongations of point symmetries.

\section{Variational Symmetries}

When $d=0$, the 2D generalized double dispersion potential Equation (10) admits a local Lagrangian structure (11) in terms of a Lagrangian functional (17), which will be used in this section to determine which of the Lie symmetries of Equation (10) with $d=0$ are variational symmetries.

A variational symmetry is an infinitesimal symmetry $\hat{\mathbf{X}}=P \partial_{v}$ that leaves invariant a Lagrangian functional $L$ up to a total divergence,

$$
\hat{\mathbf{X}} L=D_{t} \Psi^{t}+D_{x} \Psi^{x}+D_{y} \Psi^{y},
$$

where $\Psi^{t}, \Psi^{x}, \Psi^{y}$ depend on $t, x, y, v$, and derivatives of $v$. The invariance condition is usually verified by computing the left-hand side of (43) and integrating by parts the resulting expression to obtain a total divergence expression. This invariance condition is equivalent to:

$$
E_{v}\left(P E_{v}(L)\right)=0
$$

in terms of the Euler operator (12) (i.e., the variational derivative), involving only the symmetry characteristic $P$ and the Lagrangian $L[14,15]$.

Therefore, for each of the Lie point symmetries admitted by the 2D gDD potential Equation (10) with $d=0$, it is only necessary to check that the variational symmetry condition (44) is satisfied, where $L$ is the Lagrangian (17). We next summarize the results.

Theorem 4. The $2 D$ generalized double dispersion equation in potential form (10) with $d=0$ admits the variational point symmetries spanned by the time-translation symmetry (29), the space-translation symmetries 
(30) and (31), the infinite symmetry families (32), the boost symmetry (33), and the scaling-shift symmetry (37). The scaling-shift symmetry (34) is only variational for $p=\frac{4}{3}$, and the scaling-shift symmetries (35) and (36) are not variational.

\section{Conservation Laws}

A local conservation laws of the potential 2D gDD Equation (10) is a space-time divergence expression:

$$
D_{t} T+D_{x} X+\left.D_{y} Y\right|_{G=0}=0
$$

holding on the solution space of Equation (10), with $T$ and $(X, Y)$ being respectively the density and the spatial flux, which are functions of $t, x, y, v$, and derivatives of $v$. The expression $(T, X, Y)$ is called the conserved current. For any given nonlinear evolution equation, local conservation laws are important since they describe physical quantities that do not change over time within an isolated physical process.

For the potential 2D gDD Equation (10), every non-trivial conservation law (45) is equivalent to the characteristic equation $[14,15,17]$, given by:

$$
D_{t} \tilde{T}+D_{x} \tilde{X}+D_{y} \tilde{Y}=\left(v_{t t}-v_{x x}-a v_{t t x x}-b v_{x x x x}-d v_{t x x}-\left(f\left(v_{x}\right)\right)_{x}-\beta v_{y y}\right) Q
$$

holding off of the solution space of Equation (10), where $Q, \tilde{T}, \tilde{X}$, and $\tilde{Y}$ are functions of $t, x, y, v$, and derivatives of $v$. When restricted to the solution space of Equation (10), the conserved density $\tilde{T}$ and the spatial flux $(\tilde{X}, \tilde{Y})$ respectively yield $T$ and $(X, Y)$. The function $Q$ in $(46)$ is called the conservation law multiplier.

For a given equation admitting a Lagrangian structure (11), Noether's theorem states a one-to-one correspondence between variational symmetries and locally non-trivial conservation laws $[15,17]$. In terms of the variational symmetry characteristic $P$ and the conservation law multiplier $Q$, the correspondence in Noether's theorem is equivalent to the condition:

$$
P=Q
$$

Given a variational symmetry characteristic $P$, it is straightforward to derive the corresponding conserved current $(\tilde{T}, \tilde{X}, \tilde{Y})$ from the characteristic Equation (46) by using several methods. One method consists of first splitting the characteristic equation $D_{t} \tilde{T}+D_{x} \tilde{X}+D_{y} \tilde{Y}=P E_{v}(L)$ with respect to $v$ and its derivatives and then integrating the resulting linear system [17]. A second method consists of applying a repeated integration process [18] to the terms in the expression $P E_{v}(L)$ to obtain $\tilde{T}, \tilde{X}, \tilde{Y}$. A third method consists of inverting the Euler operator in the variational symmetry equation $E_{v}\left(P E_{v}(L)\right)=0$ by means of a homotopy integral formula $[14,15,17]$.

Since the 2D gDD equation in potential form (10) with $d=0$ possesses a Lagrangian formulation, we now derive the conservation laws associated with the variational symmetries obtained in Theorem 4 . 
Theorem 5. (i) The conservation laws admitted by the $2 D$ generalized double dispersion equation in potential form (10) with $d=0$, for arbitrary $f\left(v_{x}\right), a, b, \beta$, arising from variational symmetries, are given by:

$$
\begin{aligned}
& T_{1}=\frac{1}{2} a v_{t x}^{2}-\frac{1}{2} b v_{x x}^{2}+\frac{1}{2} v_{t}^{2}+\frac{1}{2} v_{x}^{2}+\frac{1}{2} \beta v_{y}^{2}+F\left(v_{x}\right), \\
& X_{1}=-a v_{t t x} v_{t}-b v_{x x x} v_{t}+b v_{t x} v_{x x}-v_{x} f\left(v_{x}\right)-v_{t} v_{x}, \\
& Y_{1}=-\beta v_{t} v_{y}, \\
& T_{2}=a v_{t x} v_{x x}+v_{t} v_{x}, \\
& X_{2}=-a v_{x} v_{t t x}-b v_{x} v_{x x x}-\frac{1}{2} a v_{t x}^{2}+\frac{1}{2} b v_{x x}^{2}+F\left(v_{x}\right)-v_{x} f\left(v_{x}\right)-\frac{1}{2} v_{t}^{2}-\frac{1}{2} v_{x}^{2}+\frac{1}{2} \beta v_{y}^{2}, \\
& Y_{2}=-\beta v_{x} v_{y} \\
& T_{3}=a v_{t x} v_{x y}+v_{t} v_{y}, \\
& X_{3}=-a v_{t t x} v_{y}-b v_{x x x} v_{y}+b v_{x x} v_{x y}-v_{y} f\left(v_{x}\right)-v_{x} v_{y}, \\
& Y_{3}=-\frac{1}{2} a v_{t x}^{2}-\frac{1}{2} b v_{x x}^{2}-\frac{1}{2} v_{t}^{2}+\frac{1}{2} v_{x}^{2}-\frac{1}{2} \beta v_{y}^{2}+F\left(v_{x}\right), \\
& T_{4}=(g(y+\sqrt{\beta} t)+h(y-\sqrt{\beta} t)) v_{t}-\sqrt{\beta}\left(g^{\prime}(y+\sqrt{\beta} t)-h^{\prime}(y-\sqrt{\beta} t)\right) v \\
& X_{4}=-(g(y+\sqrt{\beta} t)+h(y-\sqrt{\beta} t))\left(a v_{t t x}+b v_{x x x}+f\left(v_{x}\right)+v_{x}\right), \\
& Y_{4}=\sqrt{\beta}\left(\left(g^{\prime}(y+\sqrt{\beta} t)+h^{\prime}(y-\sqrt{\beta} t)\right) v-(g(y+\sqrt{\beta} t)+h(y-\sqrt{\beta} t)) v_{y}\right)
\end{aligned}
$$

where $F^{\prime}\left(v_{x}\right)=f\left(v_{x}\right)$.

(ii) The $2 D$ generalized double dispersion equation in potential form (10) with $d=0$ admits additional conservation laws corresponding to variational symmetries arising for the following special $f\left(v_{x}\right), a$, or $b$ :

(a) $a=0$, arbitrary $f\left(v_{x}\right), b$ and $\beta$,

$$
\begin{aligned}
T_{5} & =-\frac{1}{2} b y v_{x x}^{2}+\frac{1}{2} y v_{t}^{2}+\frac{1}{2} y v_{x}^{2}+\beta \frac{1}{2} y v_{y}^{2}+\beta t v_{t} v_{y}+y F\left(v_{x}\right) \\
X_{5} & =-b \beta t v_{x x x} v_{y}-b y v_{x x} v_{t}+b y v_{t x} v_{x x}+b \beta t v_{x x} v_{x y}-\beta t v_{y} f\left(v_{x}\right)-y v_{t} f\left(v_{x}\right)-\beta t v_{x} v_{y}-y v_{t} v_{x}, \\
Y_{5} & =-\frac{1}{2} b \beta t v_{x x}^{2}-\beta y v_{t} v_{y}-\frac{1}{2} \beta t v_{t}^{2}-\frac{1}{2} \beta t v_{x}^{2}-\frac{1}{2} \beta^{2} t v_{y}^{2}+\beta t F\left(v_{x}\right),
\end{aligned}
$$

where $F^{\prime}\left(v_{x}\right)=f\left(v_{x}\right)$.

(b) $f\left(v_{x}\right)=\alpha e^{p v_{x}}-v_{x}, b=0$, arbitrary $a$ and $\beta$,

$$
\begin{aligned}
& T_{6}=\frac{1}{4} a p t v_{t x}^{2}+\frac{1}{2} a p y v_{x x} v_{t y}+\frac{1}{2} a p v_{x x} v_{t}+\frac{1}{4} p t v_{t}^{2}+\frac{1}{4} \beta p t v_{y}^{2}+\frac{1}{2} p y v_{y} v_{t}+x v_{t}+\alpha t \frac{1}{2} e^{p v_{x}}, \\
& X_{6}=-\frac{1}{2} a\left(p t v_{t}+p y v_{y}+2 x\right)\left(v_{t t x}+\alpha e^{p v_{x}}\right)+\frac{1}{2} a\left(p y v_{x y}+2 a\right) v_{t t}-\frac{1}{2} a\left(p v_{t}+p y v_{t y}\right) v_{t x}, \\
& Y_{6}=-\frac{1}{2} a p y v_{t t} v_{x x}+\frac{1}{4} a p y v_{t x}{ }^{2}-\frac{1}{2} \beta p t v_{t} v_{y}-\frac{1}{4} \beta p y v_{y}^{2}-\beta x v_{y}-\frac{1}{4} p y v_{t}^{2}+\frac{1}{2} \alpha y e^{p v_{x}} .
\end{aligned}
$$

(c) $\quad f\left(v_{x}\right)=\alpha\left(v_{x}+c\right)^{7 / 3}-v_{x}, a=0$, arbitrary $b$ and $\beta$,

$$
\begin{gathered}
T_{7}=-2 b t v_{x x}^{2}+2 t v_{t}^{2}+2 \beta t v_{y}^{2}+2 x v_{t} v_{x}+4 y v_{t} v_{y}+v v_{t}+3 x c v_{t}+\frac{6}{5} \alpha t\left(v_{x}+c\right)^{10 / 3}, \\
X_{7}=-b\left(3 c x+4 t v_{t}+2 x v_{x}+4 y v_{y}+b v\right) v_{x x x}+b\left(4 t v_{t x}+x v_{x x}+4 y v_{x y}+3 c+4 v_{x}\right) v_{x x} \\
\quad-\alpha\left(\frac{12}{5} c x+4 t v_{t}+\frac{7}{5} x v_{x}+4 y v_{y}+v\right)\left(v_{x}+c\right)^{7 / 3}+\beta x v_{y}^{2}-x v_{t}^{2}, \\
Y_{7}=-2 b y v_{x x}^{2}-3 x c \beta v_{y}-4 \beta t v_{t} v_{y}-2 \beta x v_{x} v_{y}-2 \beta y v_{y}^{2}-\beta v v_{y}-2 y v_{t}^{2}+\frac{6}{5} \alpha y\left(v_{x}+c\right)^{10 / 3} .
\end{gathered}
$$

Next, we look at the meaning of these conservation laws. 
When we consider solutions $v(t, x, y)$ of the given equation in a spatial domain $\Omega \subseteq \mathbb{R}^{2}$, for every conservation law (45), there is an associated conserved integral, given by:

$$
\mathcal{C}[v]=\int_{\Omega} T d x d y
$$

satisfying:

$$
\frac{d}{d t} \mathcal{C}[v]=-\int_{\partial \Omega}(X, Y) \cdot \hat{\mathbf{n}} d s
$$

where $\hat{\mathbf{n}}$ represents the unit normal vector, which points outward to $\partial \Omega$, the boundary curve of $\Omega$, whereas $d s$ represents the arc length along this curve. The global Equation (56) physically means that there is a balance between the rate of change of the quantity (55) on $\Omega$ and the net outward flux through $\partial \Omega$.

For the 2D generalized double dispersion potential Equation (10), the conservation law (48) leads to the conserved quantity:

$$
\mathcal{E}[v]=\int_{\Omega}\left(\frac{1}{2} a v_{t x}^{2}-\frac{1}{2} b v_{x x}^{2}+\frac{1}{2} v_{t}^{2}+\frac{1}{2} v_{x}^{2}+\frac{1}{2} \beta v_{y}^{2}+F\left(v_{x}\right)\right) d x d y,
$$

where $F^{\prime}\left(v_{x}\right)=f\left(v_{x}\right)$, which is an energy arising from the time-translation symmetry (29). The conservation laws (49) and (50) yield, respectively, the conserved quantities:

$$
\begin{aligned}
& \mathcal{P}^{x}[v]=\int_{\Omega}\left(a v_{t x} v_{x x}+v_{t} v_{x}\right) d x d y, \\
& \mathcal{P}^{y}[v]=\int_{\Omega}\left(a v_{t x} v_{x y}+v_{t} v_{y}\right) d x d y,
\end{aligned}
$$

which are momentum quantities arising from the space-translation symmetries (30) and (31). The infinite family of conservation laws (51) leads to an infinite family of conserved quantities:

$$
\mathcal{T}[v]=\int_{\Omega} \sqrt{\beta}\left(\left(g^{\prime}(y+\sqrt{\beta} t)+h^{\prime}(y-\sqrt{\beta} t)\right) v-(g(y+\sqrt{\beta} t)+h(y-\sqrt{\beta} t)) v_{y}\right) d x d y,
$$

arising from the infinite symmetry families (32) and corresponding to the conserved quantities of transverse momenta of the linear wave equation $v_{t t}-\beta v_{y y}=0$. The conservation law (52) yields the conserved quantity:

$$
\mathcal{Q}[v]=\int_{\Omega}\left(-\frac{1}{2} b y v_{x x}^{2}+\frac{1}{2} y v_{t}^{2}+\frac{1}{2} y v_{x}^{2}+\beta \frac{1}{2} y v_{y}^{2}+\beta t v_{t} v_{y}+y F\left(v_{x}\right)\right) d x d y,
$$

which is a boost-momentum arising from the boost symmetry (33). The conservation laws (53) and (54) yield, respectively, the conserved quantities:

$$
\begin{aligned}
& \tilde{\mathcal{E}}_{1}[v]=\int_{\Omega}\left(\frac{1}{4} a p t v_{t x}^{2}+\frac{1}{2} a p y v_{x x} v_{t y}+\frac{1}{2} a p v_{x x} v_{t}+\frac{1}{4} p t v_{t}^{2}+\frac{1}{4} \beta p t v_{y}^{2}+\frac{1}{2} p y v_{y} v_{t}+x v_{t}+\alpha t \frac{1}{2} e^{p v_{x}}\right) d x d y \\
& \tilde{\mathcal{E}}_{2}[v]=\int_{\Omega}\left(-2 b t v_{x x}^{2}+2 t v_{t}^{2}+2 \beta t v_{y}^{2}+2 x v_{t} v_{x}+4 y v_{t} v_{y}+v v_{t}+3 x c v_{t}+\frac{6}{5} \alpha t\left(v_{x}+c\right)^{10 / 3}\right) d x d y
\end{aligned}
$$

which are dilational energy quantities arising from the scaling and shift symmetries (37) and (34).

\section{Conclusions}

For the 2D generalized double dispersion Equation (8), we first expressed this equation in potential form, and then, we obtained a condition for this equation to admit a Lagrangian formulation. We also gave the corresponding Hamiltonian structure. Next, we classified all Lie symmetries (point and contact) of the 2D gDD potential Equation (10). Finally, we constructed all conservation laws that 
arise from variational point symmetries of (10) with $d=0$. We remark that all of the conservation laws (48)-(54) of the potential Equation (10) depended essentially on the potential $v$; therefore, for the 2D gDD Equation (8), the corresponding conservation laws are nonlocal. Furthermore, we gave the physical meaning of the corresponding conserved quantities.

In future work, we will look for exact group-invariant solutions of Equation (8) by using systematically all symmetries and conservation laws of the equation. Specifically, particular cases of the generalized Equation (8) have line solitons and lump solutions, so we plan to study the line solitons and lump solution and other kinds of solitary waves of the generalized Equation (8).

Author Contributions: Conceptualization, E.R., T.M.G., R.d.1.R. and M.S.B.; methodology, E.R., T.M.G., R.d.1.R. and M.S.B.; software, E.R., T.M.G., R.d.l.R. and M.S.B.; validation, E.R., T.M.G., R.d.l.R. and M.S.B.; formal analysis, E.R.; investigation, E.R.; writing-original draft preparation, E.R.; writing-review and editing, E.R., T.M.G., R.d.l.R. and M.S.B.; supervision, E.R.

Acknowledgments: The authors thank the referees for their comments and suggestions. Stephen Anco is also gratefully thanked for the valuable remarks.

Conflicts of Interest: The authors declare no conflict of interest.

\section{Appendix A}

We provide the computational steps followed to solve the determining Equation (28) for point symmetries (26) by using the software Maple.

Firstly, we set up the determining Equation (28), and we used the command "coeffs" to split this equation with respect to the variables $\partial^{2} v, \partial^{3} v$, and $\partial^{4} v$. Note that a leading derivative of Equation (8) and its differential consequences can be previously substituted to simplify the determining Equation (28). From the leading derivatives $v_{t t}, v_{x x x x}$ or $v_{t t x x}$ of Equation (8), it is convenient to choose any of the fourth-order derivatives $v_{x x x x}$ or $v_{t t x x}$. This procedure gives an overdetermined system of 207 equations for the symmetry characteristic $P, f\left(v_{x}\right), a, b, d$, and $\beta$. We also consider the classification conditions $f^{\prime \prime}\left(v_{x}\right) \neq 0, a^{2}+b^{2} \neq 0$, and $\beta \neq 0$.

Secondly, we use the command "rifsimp" to obtain a tree containing all solution cases.

Thirdly, every solution case consists of a system of PDEs for $P$ and, possibly, an ODE for the function $f\left(v_{x}\right)$ and conditions for the parameters of the equation $a, b, d$, and $\beta$. We solve the ODE for $f\left(v_{x}\right)$ by using the command "dsolve" and the system of PDEs for $P$ by using the command "pdsolve". We check the solutions by substituting in the overdetermined system.

Finally, by applying the method detailed in the Appendix of [19], the overlapping cases are combined, giving a classification of all point symmetries admitted by the $2 \mathrm{D}$ generalized double dispersion Equation (8).

\section{References}

1. Boussinesq, J. Théorie de $\mathrm{l}^{\prime}$ intumescence liquide appelée 'onde solitaire' ou 'de translation', se propageant dans un canal rectangulaire. C. R. Acad. Sci. 1871, 72, 755-759.

2. Boussinesq, J. Théorie des ondes et des remous qui se propagent le long d'un canal rectangulaire horizontal, en communiquant au liquide continu dans ce canal des vitesses sensiblement pareilles de la surface au fond. J. Math. Pure Appl. 1872, 17, 55-108.

3. Chen, G.; Wang, Y.; Wang, S. Initial boundary value problem of the generalized cubic double dispersion equation. J. Math. Anal. Appl. 2004, 299, 563-577. [CrossRef]

4. Samsonov, A.M.; Sokurinskaya, E.V. Energy Exchange between Nonlinear Waves in Elastic Waveguides and External Media Nonlinear Waves in Active Media; Springer: Berlin, Germany, 1989.

5. Wang, S.; Chen, G. Cauchy problem of the generalized double dispersion equation. Nonlinear Anal. 2006, 64, 159-173. [CrossRef]

6. Yacheng, L.; Runzhang, X. Potential well method for Cauchy problem of generalized double dispersion equations. J. Math. Anal. Appl. 2008, 338, 1169-1187. 
7. Zhengde, D.; Boling, G. Long time behavior of nonlinear strain waves in elastic waveguides. J. Part. Diff. Eq. 1999, 12, 301-312.

8. Zhengde, D.; Xianyun, D. Global attractor for the nonlinear strain waves in elastic waveguides. Acta Math. Sci. 2000, 17, 260-270. [CrossRef]

9. Wang, S.; Esfahani, A. Global rough solutions to the sixth-order Boussinesq equation. Nonlinear Anal. 2014, 102, 97-104. [CrossRef]

10. Recio, E.; Gandarias, M.L.; Bruzón, M.S. Symmetries and conservation laws for a sixth-order Boussinesq equation. Chaos Solitons Fractals 2016, 89, 572-577. [CrossRef]

11. Anco, S.C.; Gandarias, M.L.; Recio, E. Conservation laws, symmetries, and line soliton solutions of generalized KP and Boussinesq equations with $p$-power nonlinearities in two dimensions. Theor. Math. Phys. 2018, 197, 1393-1411. [CrossRef]

12. Matsukawa, M.; Watanaba, S.; Tanaca, H. Soliton solutions of generalized 2D Boussinesq equation with quadratic and cubic nonlinearity. J. Phys. Soc. Jpn. 1989, 58, 827-830. [CrossRef]

13. Rui, W.; Zhao, P.; Zhang, Y. Invariant solutions and conservation laws of the $(2+1)$-dimensional Boussinesq equation. Abstr. Appl. Anal. 2014, 840405. [CrossRef]

14. Anco, S.C. Generalization of Noether's theorem in modern form to non-variational partial differential equations. In Fields Institute Communications: Recent progress and Modern Challenges in Applied Mathematics, Modeling and Computational Science; Springer: New York, NY, USA, 2017; pp. 119-182.

15. Olver, P.J. Applications of Lie Groups to Differential Equations; Springer: New York, NY, USA, 1993.

16. Anco, S.C.; Bluman, G. Direct construction method for conservation laws of partial differential equations Part II: General treatment. Eur. J. Appl. Math. 2002, 41, 567-585. [CrossRef]

17. Bluman, G.W.; Cheviakov, A.; Anco, S.C. Applications of Symmetry Methods to Partial Differential Equations; Springer: New York, NY, USA, 2009.

18. Wolf, T. A comparison of four approaches to the calculation of conservation laws. Eur. J. Appl. Math. 2002, 13, 129-152. [CrossRef]

19. Recio, E.; Anco, S.C. Conservation laws and symmetries of radial generalized nonlinear $p$-Laplacian evolution equations. J. Math. Anal. Appl. 2017, 452, 1229-1261. [CrossRef]

(C) 2019 by the authors. Licensee MDPI, Basel, Switzerland. This article is an open access article distributed under the terms and conditions of the Creative Commons Attribution (CC BY) license (http:/ / creativecommons.org/licenses/by/4.0/). 